\title{
Human stool preservation impacts taxonomic profiles in 16S rRNA gene-based metagenomics studies
}

\section{Anne Plauzolles ( $\nabla$ a.plauzolles@alphabio.fr )}

AlphaBio https://orcid.org/0000-0001-6076-8626

Eya Toumi

Alphabio

Benoit Goutorbe

Alphabio

Marion Bonnet

AlphaBio

Guillaume Pénaranda

Alphabio

Ghislain Bidaut

CRCM: Centre de Recherche en Cancerologie de Marseille

\section{Laurent Chiche}

Hôpital Européen Marseille

Jérôme Allardet-Servent

Hôpital Européen Marseille

Frédérique Retornaz

Hôpital Européen Marseille

Philippe Halfon

Alphabio

\section{Methodology}

Keywords: microbiota, standardization, 16S rRNA gene, metagenomics, human gut, preservation, stool, stabilizing solution

Posted Date: October 9th, 2020

DOI: https://doi.org/10.21203/rs.3.rs-87437/v1

License: (a) (i) This work is licensed under a Creative Commons Attribution 4.0 International License.

Read Full License 



\section{Human stool preservation impacts taxonomic profiles in $16 \mathrm{~S}$}

\section{2 rRNA gene-based metagenomics studies}

3

4 Anne Plauzolles $^{1 \dagger}$, Eya Toumi ${ }^{1 \uparrow}$, Benoit Goutorbe ${ }^{1,2}$, Marion Bonnet ${ }^{1}$, Guillaume Pénaranda ${ }^{1}$,

5 Ghislain Bidaut ${ }^{2}$, Laurent Chiche ${ }^{3}$, Jérôme Allardet-Servent ${ }^{4}$, Frédérique Retornaz ${ }^{3}$, Philippe

6 Halfon ${ }^{1,3}$.

7

8

$9 \quad{ }^{1}$ Clinical Research and R\&D Department, Laboratoire Européen Alphabio, 13003 Marseille, France

${ }^{2}$ CRCM, Aix-Marseille Univ U105, Inserm U1068, CNRS UMR7258, Institut Paoli-Calmettes, Marseille, France

${ }^{3}$ Infectious and Internal Medicine Department, Hôpital Européen Marseille, 13003 Marseille, France

${ }^{4}$ Intensive care unit, Hôpital Européen Marseille, Marseille, France

${ }^{\dagger}$ The two authors contributed equally to this work. 
Background: Microbiotas play critical roles in human health, yet in most cases scientists lack standardized and reproducible methods. To date, stool sample preservation remains a source of technological bias in metagenomic sequencing, despite newly developed storage solutions. Studying those biases will help in identifying the appropriate stool preservation approach to promote the implementation of standard operating procedure and improve comparability across multiple microbiome studies.

Results: Here, we conducted a comparative study of 10 storage methods for human stool over a 15-day period of storage at fluctuating temperatures. We first compared the performance of each stabilizer with observed bacterial composition variation within the same specimen. Then, we identified the nature of the observed variations to determine which bacterial populations were more impacted by the stabilizer. We found that DNA stabilizers display various stabilizing efficacies and affect the recovered bacterial profiles. Furthermore, our results showed that the bias associated with the stabilizers can be linked to the phenotypical characteristics of the bacterial populations present in the studied samples.

Conclusions: Although newly developed storage solutions have improved our capacity to stabilize stool microbial content over time, they are nevertheless not devoid of biases. We therefore recommend considering the nature of these technological biases before claiming whether some microbes are beneficial or even deleterious to human health.

\footnotetext{
Keywords : microbiota, standardization, 16S rRNA gene, metagenomics, human gut,
} preservation, stool, stabilizing solution 


\section{Background}

42

43

Over the past decade, an increasing number of studies have been published focusing on the human microbiome. While there is no doubt that these findings have helped us better comprehend the complexity of our microbiome and its implications on our health, the knowledge accumulated in the microbiome field is not equivalent to the amount of research and effort provided by the scientific community. The lack of standards hampers our expertise, as studies show inconsistencies, often resulting from technological bias rather than a true biological signature. To utilize microbiome science to its full potential, technical and computational methods must be standardized, and quality controls must be implemented to transition in the near future from a basic research environment to the clinic.

It is now common knowledge that our microbiome colonizes all body surfaces, especially our gut microbiome, which strongly impacts nearly every aspect of host physiology [1-3].

Multiple lines of evidence now link alterations in the gut microbiome to numerous diseases $[2,4-8]$. However, microbiome studies most often lead to mixed results, halting our progress and hindering potential diagnosis, disease prediction and therapeutic intervention of microbiome analyses. Hence, no individual bacteria are consistently associated with a given disease. Such discrepancies, in regard to microbiome signature patterns, are likely due to heterogeneity across study populations (small size, genetic factors, lifestyle) or the studied model or could be influenced by methodological differences among studies [9-11].

The reality of microbiome research is that a variety of biological and technical factors can impact the quality of samples and their microbial content [12]. The gut microbiome is the most challenging human ecosystem to characterize due to its heterogeneous bacterial populations. Its composition varies widely from one individual to another and involves a majority of bacterial populations that are very sensitive to oxygen [13], as well as remnants of 
human and food DNA and inhibitors likely to hamper subsequent analytical steps [14]. Technical bias can then result in misleading findings and can affect the quality of the data. Throughout the series of steps that a fecal sample undergoes to identify and characterize its microbial content, sampling and stabilization are key in the preanalytical protocol and can heavily impact data quality. Previous studies have demonstrated that storage conditions of stool samples have only a small impact on their microbial content [15-16]; however, more recent findings show otherwise [17-21]. DNA and RNA deteriorate rapidly after collection when kept at room temperature [17], while the chemistry of existing stabilizing solutions has also demonstrated an impact on the recovery of genomic microbial content, resulting in a source of bias $[22,23]$. Despite these conflicting results and challenges, a few principles are currently well acknowledged by the scientific community: avoid freeze-thaw cycles and temperature fluctuations throughout the preservation process $[12,17,20,24]$; when possible, shorten the transportation time; and freezing samples at $-20^{\circ} \mathrm{C}$ or $-80^{\circ} \mathrm{C}$ provides an optimal solution when immediate analysis of fresh sample is not an option [20, 21, 23, 25-33].

In regard to studying microbiome composition using metagenomics, the method of collection that yields the most accurate results involves analyzing samples immediately after collection. However, this can be logistically challenging for samples such as stools that cannot be produced on demand. Any stabilizing method induces rapid changes in the presence and/or abundance of certain bacterial populations [27]. Despite different efficacies in stabilizing the true biological profile, the preservation step can result in biases even during short-term storage, but these alterations are, for most commonly utilized solutions, smaller or comparable to differences among technical replicates. Technical variability, albeit smaller than interindividual variability, may obscure subtle and meaningful alterations. Therefore, the choice of stabilization is highly dependent on factors such as limitations, availability, ease of use, cost and compatibility with the study's goals and/or 'omic' methods. 
While the lack of standards affects the microbiome field in every 'omic' science and their related testing phases, including preanalytical, analytical and postanalytical steps in sample processing, our research here focuses on technical bias in the preanalytical handling of fecal samples in the study of gut microbiota through $16 \mathrm{~S}$ rRNA gene based metagenomics analysis. For the past few years, the lack of standards and the sources of errors in datasets have been highlighted in the literature. Emerging protocols have arisen, but comparative studies, including comparison of most recent DNA stabilizers, are lacking. Our study aimed to evaluate and compare a large panel of stabilizing solutions that are either widely used by the scientific community or suited to the collection of fecal material. We also investigated the dynamic alterations that occurred over time in our samples based on their bacterial content and related phenotypical characteristics. Based on these results, acknowledging and identifying the limitations of DNA preservation could promote comparability among metagenomics studies and lead to clear guidelines that will be critical for scientific discovery going forward in understanding human microbiomes.

\section{METHODS}

\section{Stool conservation study design}

To provide a standardized protocol for fecal sampling and preservation, fecal samples were collected from 15 French volunteers $(n=15)$.

To evaluate the interaliquot variability, 3 aliquots of $180-220 \mathrm{mg}(\mathrm{Sc})$ of each fecal sample were first created and stored at $-20^{\circ} \mathrm{C}$. Fecal homogenization was then performed as follows: $12 \mathrm{~g}$ of stool was gently mixed with $30 \mathrm{ml}$ of ultrasterile water for a few minutes. This step was performed to limit variability among aliquots, allowing for better evaluation of the impact 
of the stabilizing solutions tested. The homogenized stool was then subsampled into triplicate samples of $0.5 \mathrm{ml}$ and immediately frozen at $-20^{\circ} \mathrm{C}$ without any additives (Dc) in 10 aliquots of $0.5 \mathrm{ml}$ each preserved with addition of $1 \mathrm{ml}$ of DNA stabilizer. A total of 10 stabilizing solutions were tested: RNAlater (Ambion, Austin, US), Tris-EDTA (10 mM Tris-HCl pH 8.0, 1 mM EDTA) (Thermo Fisher Scientific, Massachusetts, US), 95\% ethanol (VWR international, Pennsylvania, US), PrimeStore MTM (Longhorn Vaccines and Diagnostics, San Antonio, US), Stratec (Stratec Molecular GmbH, Berlin, Germany), OMNIgene-Gut (DNA Genotek, Ontario, Canada), Norgen (Norgen Biotek Corp., Thorold, Canada), DNA/RNA Shield (Zymo Research, Freiburg, Germany), Fecal Swab (Copan Italia S.P.A., Brescia, Italy), and Whatman FTA card (GE Healthcare Life Sciences, Illinois, US). For the FTA card method, a $0.5 \mathrm{ml}$ sample was dispatched directly on the card. All stabilizers were tested on 15 fecal samples, except for PrimeStore MTM solution, which was tested on only 13 samples. In parallel, two unstabilized aliquots were produced: one solid (180-220 mg of stool) (S) and one homogenized $(0.5 \mathrm{ml})$ (D). These two aliquots constituted controls for evolution of the microbiota without preservation.

Finally, all aliquots were preserved over a period of 15 days (Figure 1). Briefly, nonfrozen aliquots (S, D and aliquots 1 to 10 ) were incubated for 15 days at varying temperatures fluctuating from $4^{\circ} \mathrm{C}$ to $40^{\circ} \mathrm{C}$ according to the following cycle: 3 days at room temperature (RT, approximately $25^{\circ} \mathrm{C}$ ), 3 days at $4^{\circ} \mathrm{C}, 3$ days at $\mathrm{RT}, 3$ days at $40^{\circ} \mathrm{C}$ and 2 days at $\mathrm{RT}$. These temperature fluctuations allowed evaluation of the efficacies of each stabilizing solutions in harsh conditions. The temperature range chosen includes temperatures that a sample can be subjected to during transportation to the laboratory throughout the seasons for most countries worldwide.

The evolution of all samples was compared to the baseline samples (i.e., homogenized and immediately frozen samples stored with no additives, Dc). 


\section{Stool DNA extraction}

141 Bacterial DNA was isolated from all stool aliquots using the NucleoSpin ${ }^{\circledR}$ DNA Stool kit 142 (Macherey-Nagel, Duren, Germany) following the manufacturer's instructions. Extracted 143 DNA was stored at $-20^{\circ} \mathrm{C}$ until subsequent application.

\section{DNA quantification and purity measurements}

DNA quantification and purity (A260/A280 ratio) measurements were performed by spectrophotometry using a Nanodrop ND-1000 (Thermo Fisher Scientific, Massachusetts, US).

16S rRNA gene amplification, library preparation and high-throughput

\section{sequencing}

To determine the bacterial composition of each aliquot, a 16S metagenomic sequencing library was created following Illumina's recommendations [34]. Briefly, this protocol targets the V3-V4 regions of the 16S rRNA gene during a first PCR using specific primers with overhang adapters: 16S Amplicon PCR Forward Primer 5'

TCGTCGGCAGCGTCAGATGTGTATAAGAGACAGCCTACGGGNGGCWGCAG and 16S Amplicon PCR Reverse Primer 5' 
Index Kit (Illumina, San Diego, US). Following a second purification with Agencourt

AMPure XP magnetic beads, the PCR products were then checked with quality controls using a fragment analyzer (Agilent Technologies, California, US) and Qubit (Thermo Fisher Scientific, Massachusetts, US) to evaluate DNA fragment sizes and DNA concentrations of the purified products. Barcoded amplicons were pooled in equal concentrations to generate a $4 \mathrm{nM}$ library. The pool of samples was denatured to a final concentration of $12 \mathrm{pM}$ and combined with 5\% PhiX control (Illumina, San Diego, US). The 16S rRNA gene libraries were sequenced using a MiSeq instrument (Illumina, San Diego, US).

\section{Experimental validation}

Demultiplexed and high-quality sequences (average quality score $>$ Q30) were retrieved. All samples below 40,000 reads were discarded. A total of six aliquots, five stabilized with Stratec solution, were excluded due to low quality DNA, which is further discussed below (see Results section). A clustering analysis was performed on the results to validate the experiment. Three samples were excluded from our analysis as they did not cluster with their technological replicates (Supplementary Figure S1 for details).

\section{Bioinformatics processing}

Reads were processed using QIIME 2 [35] (version 2019.1.0) and its DADA2 [36] plugin (q2dada2, version 2019.1.0). Preprocessing parameters were tuned to our dataset's specifications: reads were trimmed at their 3' ends at $245 \mathrm{bp}$, and reads shorter than this threshold were discarded; to remove amplification primers, 5' trimming was performed at 17 bp and $21 \mathrm{bp}$ for forward and reverse reads, respectively. Reads that exceeded the 2 sequencing errors expected were discarded, and chimera removal was performed with the consensus method of DADA2. A denoising step was performed, and amplicon sequence 
variants (ASVs) were collected in a counting table. Taxonomic assignment was performed using Kraken [37] based on the NCBI RefSeq Targeted Loci database, which contains over 21,000 bacterial and archaeal 16S reference sequences covering more than 15,000 species.

\section{Statistical analysis}

Statistical analysis was performed with R (version 3.4) using the phyloseq package [38] (version 1.22.3). Beta diversity was assessed with several metrics: Jaccard and Bray-Curtis dissimilarity indices were computed based on rarefied data, while Aitchison's distances [39] were computed based on centered log-ratio transformed data with pseudo counts set at 0.5 . Only the Bray-Curtis based analysis is shown, but different metrics confirmed this result (Supplementary Figures S2, S3 and S4).

First, the impact of homogenization was assessed by comparing mean distances within technological Sc (not homogenized) and Dc (homogenized) replicates across all stool samples collected using a paired Wilcoxon test.

Second, we evaluated stabilization performance by measuring the distance between each sample and its reference, defined as the barycenter of the 'Dc' replicates for the corresponding stool sample. We used a Kruskal-Wallis test to highlight the effect of the stabilizing solution on preservation of the bacterial content over storage time. Afterward, we performed a pairwise paired Wilcoxon test with Benjamini-Hochberg p-value correction for multiple hypothesis testing to determine which solutions performed better than others.

Finally, we searched for differentially abundant taxa, at the phylum and genus levels, between reference and stabilized samples with a Wilcoxon test, and p-values were adjusted with the Benjamini-Hochberg procedure. Furthermore, we gathered phenotypic data for the top 50 genera, representing up to $94 \%$ of all organisms found, regarding their oxygen sensitivity and their Gram stain status, as these characteristics are often conserved at the genus level [40-42]. 
We used the LPSN database [43] to identify reference articles describing the characteristics of

213 each genus. Gram stain status was defined as positive, negative or variable, and oxygen sensitivity was defined as strictly aerobic, strictly anaerobic, facultative anaerobic or microaerophile. Data are provided in Supplementary Table T1. We then clustered genera based on their median $\log 2$-fold change between stabilized samples and references using L2 distances and Ward's linkage to identify genera that behaved similarly in the stabilizing solutions tested. To track potential links between genus phenotype and behavior in stabilizing solutions, we performed a $\chi^{2}$ test for independence of categorical variables between genera clusters and both oxygen sensitivity and Gram stain status independently. To further investigate these links, we aimed to determine whether phenotypic characteristics could prelude the emergence of the storage bias that we observed. Therefore, for each solution, we performed a Kruskal-Wallis test among genera for log2-fold change and both oxygen sensitivity and Gram stain status independently.

\section{RESULTS}

In the present study, the performance of each stabilizer was defined as the microbial community alterations over time relative to the baseline sample (i.e., immediately frozen sample stored with no additive). The technical reproducibility of our analytical protocol was evaluated using triplicates of baseline samples, while samples with no additive (S and D) served as indicators of the natural evolution of the microbiota profile if unstabilized. 
Analysis of complex microbial ecosystems requires high-quality libraries for next generation sequencing (NGS) metagenomics. Hence, preserving a microbial profile over time and providing good DNA yield and purity of DNA extracts are key aspects in the analytical protocol in place. We found considerable differences in the DNA concentrations and A260/280 ratios of our extracted DNA. For example, Fecal Swab-preserved samples recovered, on average, 12-fold more DNA than Stratec-preserved samples $(60.28 \mathrm{ng} / \mu \mathrm{lvs}$ $4.97 \mathrm{ng} / \mu \mathrm{l})$. Among the different stabilizers tested in this study, recovered DNA was the lowest for Stratec-, DNA/RNA Shield- and FTA card-stabilized samples (Figure 2a). In addition, samples preserved with these three solutions had primarily low A260/280 ratios (mean ratio <1.7), indicating the presence of contaminants (Figure 2b). Interestingly, Stratec samples were the least successful for recovering a microbiota profile with sufficient reads and showed a smaller alpha diversity than other stabilizers, while DNA/RNA Shield- and FTA card-preserved samples exhibited good profile recovery with high alpha diversity values (Supplementary Figure S1). The diversity index showed similar alpha diversity among preservation methods, and the Stratec stabilizer presented a much lower alpha diversity measure than the other stabilizers. As such, these results do not show any relationships among DNA concentration/purity, diversity and microbial profile recovery.

Dc and Sc measures resulted in similar concentrations and quality ratios among triplicate samples. Unstabilized samples (Dc, Sc, D and S) showed the highest DNA yield as they recovered on average 2.6-fold more DNA than stabilized samples with good A260/280 ratios.

A total of six samples were discarded, including five Stratec-stabilized samples and one Dc sample due to a lack of compliance with quality and/or quantity criteria. 
Homogenization is commonly performed in studies to minimize intrasample variations and subsequent misestimation of the observed alterations within recovered profiles. The interaliquot variability for each Sc and Dc triplicate was first estimated by the mean distance using several methods (Bray-Curtis distance, Jaccard distance, and Aitchison distance).

Comparison of distances within triplicates and between Sc and Dc triplicates showed a greater dispersion in $\mathrm{Sc}$ than in Dc triplicates, regardless of the distance method used (Figure 3 and Supplementary Figure S2). In addition, a Wilcoxon test showed that homogenization significantly reduced observed interaliquot variability $(\mathrm{p}=0.02557)$.

These results suggest that stool subsampling results in variations in the recovered microbial content among aliquots and confirms that homogenization of each sample has contributed here to significantly lowering the interaliquot variability. In this study, our homogenized aliquots added to the different stabilizers can thus be considered identical prior to storage. Their evolution over the 15-day storage period then provides an adequate evaluation of the efficacy of each stabilizer tested when compared to their reference (i.e., an average of Dc triplicates).

\section{DNA stabilizers alter stool microbial composition with various magnitudes}

\section{compared to samples with no additives}

To evaluate the performance of the tested stabilizers, we quantified the compositional dissimilarity between each preserved sample and its reference, defined as the barycenter of the 'Dc' replicates. Different metrics, including the Bray-Curtis, Jaccard, and Aitchison distances, show that Norgen, DNA/RNA Shield, OMNIgene-Gut and PrimeStore MTM produced profiles closest to their reference, while the remaining stabilizers resulted in greater alterations (Figure 4A and Supplementary Figures S3A and S4A). A Kruskal-Wallis test $(\mathrm{p}<10-11)$ then confirmed that the solutions tested demonstrated distinct efficacies of 
stabilization specific to each stabilizer. Finally, a paired Wilcoxon test was used to compare the stabilizing performance among all stabilizers tested and identified Norgen as the best performing solution, closely followed by OMNIgene-Gut, DNA/RNA Shield and PrimeStore MTM, which presented similar performances (Figure 4B and Supplementary Figures S3B and S4B). In contrast, the least efficient stabilizers were Stratec, FTA card and Tris-EDTA, which appear no better than unstabilized samples (S or D).

In parallel, the results suggested that interindividual variability largely exceeded interaliquot variability (Figure 4A, Supplementary Figures S3A and S4A). Distances towards the reference were larger than interaliquot distances but smaller than those for interindividual variability, indicating that preservation-induced effects were observed but were smaller than biological interindividual variability. The only exception was Stratec-preserved samples, which displayed a variability similar to that observed among samples, confirming that this solution is not suitable for storage of human fecal samples. These results were confirmed by hierarchical clustering as shown in Supplementary Figure S5.

\section{Bacterial relative abundance differs based on the method of preservation in}

\section{different taxonomic ranks}

Our analysis demonstrated that bacterial taxa were affected by the stabilizer, with misestimation of their relative abundance compared to their reference profiles (Dc). These alterations were detected at different taxonomic levels, including phyla (Figure 5) and genera (Supplementary Figure S6). Observed biases specific to each stabilizing solution were statistically confirmed by a paired Wilcoxon test, which showed that regardless of their efficiency of preserving a true microbiota profile, the different solutions tested impacted the relative abundance of certain bacterial taxa recovered when a fecal sample had been stored in a stabilizer. Low-abundance phyla $(<1 \%)$, such as Tenericutes, Synergistetes and 
Verrucomicrobia, were the least significantly altered, except for Lentisphaerae, which was significantly overestimated in most storage conditions tested. Among abundant phyla (>1\%), the most significantly affected were Actinobacteria and Proteobacteria, which tended to be overestimated, while Firmicutes and Bacteroidetes were underestimated. Of all abundant phyla, Bacteroidetes were interestingly the least significantly altered.

Parallel samples that were not exposed to any additive (S, D) also showed profile alterations, suggesting an effect of storage temporality, likely due to both bacterial growth for some populations and bacterial death for others. The lack of stabilization at fluctuating temperatures resulted in significant alterations of Firmicutes, Proteobacteria, Actinobacteria and Lentisphaerae.

Among the solutions with the greatest performances for stabilizing the fecal microbiota, Norgen did not significantly alter any phyla, except for Lentisphaerae, which were overestimated. In contrast, significant alterations were observed with OMNIgene-Gut and DNA/RNA Shield, both of which significantly affected Firmicutes, Bacteroidetes, Proteobacteria and Lentisphaerae, while Actinobacteria was only affected by DNA/RNA Shield. Interestingly, PrimeStore MTM appeared to significantly disturb only Firmicutes and Lentisphaerae. Stratec, which was the least efficient for preserving the fecal microbiota in our study, seemed to only affect Actinobacteria, but this result is biased, as many Stratecpreserved samples were excluded from this analysis due to poor quality DNA and/or low read numbers compared to the other stabilizing methods.

Considering the diversity of populations that can be found within phyla and the possibility that some phenotypic characteristics may dictate or facilitate certain alterations, it is interesting to observe changes at a lower taxonomic range. Here, genera represented as $16 \mathrm{~S}$ metagenomic sequences provided reliable data up to this taxonomic rank. Genera clusters, based on alterations in the microbiota profile across all solutions tested, were found to be 
dependent on both oxygen sensitivity $(\mathrm{p}=0.0001)$ and Gram stain status $(\mathrm{p}=0.049)$ among the genera retrieved (Supplementary Figure S6 and Supplementary Table ST1). These results indicate that the physiological traits examined could potentially prelude which populations are susceptible to alteration by the DNA stabilizers. For each solution, we tested the effect of these phenotypical characteristics on the $\log 2$ fold change between a stabilized sample and its reference. We found no significant effect of oxygen sensitivity or Gram stain status on genus alterations in the absence of stabilizing solutions (samples S and D). In contrast, for stabilized samples, we found that genus alteration during storage was influenced by their oxygen status for Tris-EDTA $(\mathrm{p}=0.025)$ and in FTA card $(\mathrm{p}=0.029)$. Similarly, we found that Gram stain status affected samples stabilized with DNA/RNA Shield $(\mathrm{p}=0.002)$, PrimeStore MTM $(\mathrm{p}=0.027)$ and Stratec $(\mathrm{p}=0.043)$.

\section{DISCUSSION}

To the best of our knowledge, this study is the first in the microbiome field to compare such a large panel of storage methods, allowing identification of the best performing DNA stabilizers for a given ecosystem. We have shown that, of all stabilizers tested, some drastically impact the observed microbial composition and introduce biases. To proceed, we chose to evaluate methodologies already in use in the microbiome field through a comparative study of 10 storage methods to identify optimal fecal sampling methods that provide reproducible, stable, and accurate results.

Our analysis identified Norgen, OMNIgene-Gut, DNA/RNA Shield and PrimeStore MTM as the most efficacious stabilizers. According to our results, several comparative studies have identified OMNIgene-Gut as a good DNA stabilizer for microbiome studies [18, 27, 44, 45], 
while the other three solutions have not yet been extensively evaluated by comparative studies. In contrast, the remaining solutions tested were less efficient, showing a profile with alterations similar to unstabilized samples ( $\mathrm{S}$ and $\mathrm{D}$ ). Interestingly, among the stabilizers that were less reliable in our analysis, most showed discordant results in their ability to preserve fecal samples throughout comparative studies. For example, RNAlater was until recently the most commonly used buffer for metagenomic studies [14, 17, 18, 24, 27, 46, 47]. However, its suitability for microbiome analysis has been extensively reviewed, as some studies claim that it results in reduced overall DNA yields and reduces the detection/abundance of bacterial taxa $[18,20,21,25,26,47]$. Our results did not show reduced DNA yield compared to other preserved solutions but did show significant alterations in the recovered microbiota compared to their references, thus agreeing with previous studies that RNAlater is not an optimal preservation method. We came to the same conclusion for FTA card as Hale et al. [25], who demonstrated that FTA card (and RNAlater)-preserved samples were the least similar to fresh samples, while in contrast, Sinha et al. [26] recommended the use of FTA card for short-term storage, demonstrating that it provides reproducible, stable, and accurate data across laboratories (over 4-day storage). The longer storage time in our protocol might have contributed to our discordant results. Similar to numerous studies performing homogenization of fecal samples [18, 26-28, 33, 48, 49], homogenization of our samples contributed to a better evaluation of the true performance of each stabilizing solution for preserving the microbiota content over time, as each aliquot presented a similar profile when added to the stabilizer.

Despite various effective methods for preserving a true microbiota profile over storage time, the alterations observed between the reference samples and their 15-day-stabilized aliquots were smaller than the differences between samples (subjects), except for Stratec-preserved samples. Furthermore, triplicates for each stool sample collected did not cluster by 
preservation method. Therefore, the human gut appears to be highly subject-specific, as our results suggest that interindividual variation accounts for the major of differences observed in fecal samples and outweighs the effect (or bias) of collection and storage, as previously demonstrated in several studies $[18,19,26,27,29]$. As stated above, the only exception was Stratec-preserved samples, which displayed variability similar to that observed among samples, indicating that this solution is not suitable for storage of human fecal samples. This result contradicts a recent study [22], which concluded that the Stratec solution was a suitable storage buffer for fecal specimen preservation. However, this study was performed on a small cohort $(n=4)$ over a 7 -day period of storage at room temperature. The fluctuating temperatures in our protocol and the longer period of storage might explain the discrepancies between these findings. Additionally, our results did not demonstrate any relationships among DNA concentration/purity, microbial diversity, and microbial composition, similar to previous studies $[25,50]$. However, it has been suggested that high DNA concentrations might favor the identification of rare populations [18, 20, 25, 47]. Although the low DNA yield observed with Stratec-stabilized samples might not entirely explain the difficulties in recovering a good microbiota profile, this factor may have contributed to its poor performances in our protocol.

Finally, microbiome comparative studies investigating the effect of storage often examine variations in the relative abundances of phyla and genera specific to the stabilizing methods. However, they do not examine these alterations based on microbial population characteristics, with the literature showing that bacteria within a genus share the same general phenotypic characteristics, in particular oxygen sensitivity and Gram stain status [40-42]. In this study, we demonstrated that altered dynamics resulting from sample preservation are dictated by the phenotypical characteristics of the bacterial populations present in the studied sample. Our samples showed that genera alteration during storage is influenced by oxygen status for the Tris-EDTA and FTA card methods, as well as the Gram stain status for the DNA/RNA 
Shield, PrimeStore MTM and Stratec methods. A recent study also demonstrated that Gram status can alter the microbial content when Norgen stabilizer is used [51]. Hence, preservation of the microbiota profile is impacted by the stabilizer chosen and its efficacy for preserving the true microbial profile. However, it must be taken into consideration that the stabilizer's performance can also be affected by the microbial content of the studied sample and its most common phenotypical traits.

One limitation of our study is that we did not evaluate the stabilizing performances of each solution tested across different times or over long-term storage periods. Indeed, Sinha et al. [26] found that incubation at room temperature over 4 days reduced the reproducibility for most sampling methods, including no additives, swab, 70\% ethanol, and EDTA. As such, the performance measures in our study only reflect their efficacies over a period of 15 days throughout various temperature fluctuations but do not attest of the loss of technical reproducibility or the impact on the alteration of bacterial taxa if samples are incubated in their stabilizers for a longer period. Finally, our study only analyzed the human gut ecosystem of a French cohort. Further studies will be required in order to provide the scientific community with a more comprehensive analysis of stabilizing methods throughout different cohorts and with different types of samples to establish guidelines that will help scientists in their experimental settings.

We anticipate that procedures for microbial preservation will likely further improve in the future, and we show with this study that preservation remains a key step that can introduce technical bias into the study of complex ecosystems such as the human gut. Here, we demonstrated that some stabilizers are not suitable for the preservation of a stool sample when the sample is intended to describe the whole complexity of the human gut ecosystem through 16S metagenomics. Our data identified Norgen, OMNIgene-Gut, DNA/RNA Shield and PrimeStore MTM as the most effective stabilizers, as they resulted in reduced technical 
biases. Acknowledging the performances of stabilizing solutions and their suitability depending on the microbial content of the ecosystem studied will help establish standards in omics studies. If implemented within metagenomics protocols across laboratories, these solutions could promote experimental reproducibility among research groups and lead to meaningful knowledge about the gut microbiome and its impact on human health with the discovery of new health-associated microbiome patterns and biomarkers.

\section{CONCLUSIONS}

The diversity and complexity of the human gut microbiota increase the difficulty of elaborating a method to study such ecosystems without experimental biases. Storage conditions can introduce substantial changes to microbial community profiling in regard to $16 \mathrm{~S}$ metagenomics. Acknowledging the biases and limitations of the implemented method is key to better interpret and support true health (disease)-associated microbiome patterns that will then lead us towards personalized medicine, in which the microbiota profile could constitute a reliable tool for clinical practice.

\section{LIST OF ABBREVIATIONS}

ASVs: amplicon sequence variants

D: diluted sample

Dc: diluted sample, frozen

NGS: next generation sequencing

RT: room temperature

S: solid sample 


\section{DECLARATIONS}

\section{Ethics approval and consent to participate}

463 Fecal samples were collected from 15 volunteers. Most samples $(n=10)$ were collected in the 464 laboratory and handled immediately after defecation, while a minority $(n=5)$ were collected at 465 home and returned to our laboratory within 3 hours postdefecation. No medical records were collected. The sampling date was the only information provided by each volunteer. All subjects provided written informed consent prior to participating in the study.

\section{Consent for publication}

Not applicable. This study didn't collect any individual person's data in any form (including

471 any individual details, images or videos).

472

\section{Availability of data and material}

474 The data for this study have been deposited in the European Nucleotide Archive (ENA) at

475 EMBL-EBI under accession number PRJEB40569

476 (https://www.ebi.ac.uk/ena/browser/view/PRJEB40569). Scripts are available at

477 https://gitcrcm.marseille.inserm.fr/goutorbe/stool-preservation .

\section{Competing interests}

480 The authors declare no competing financial interests. 
$482 \quad$ Funding

483 This research received no specific grant from any funding agency in the public, commercial, or not-

484 for-profit sectors. Alphabio laboratory funded this study.

\section{Authors' contributions}

487

488

489

490

491

492

493

494

495

496

497

498

499

500

501

502

AP designed the study, extracted and sequenced samples, interpreted the results, and wrote and finalized the manuscript. ET extracted and sequenced samples and drafted the manuscript. BG performed bioinformatics analysis under the supervision of GB and statistical analysis under the supervision of GP, and drafted the manuscript. MB extracted and sequenced samples. PH finalized the manuscript and funded this study. All authors discussed the results and commented on the manuscript.

\section{Acknowledgements}

We thank our collaborators, Alphabio's molecular biology team, the CRCM Integrative Bioinformatics platform and Bernard Chetrit from the DataCentre for IT and Scientific Computing (Disc) platform for their support and involvement in the management of this study. We also thank Mahendra Mariadassou for his review of the statistical analysis.

\section{FIGURES}

Figure 1: Illustration of the experimental protocol. 
Evaluation of ten commercial DNA stabilizing solutions for the storage of fecal samples. (Sc: frozen solid sample, Dc: frozen homogenized sample, S: solid sample without stabilizer, D: homogenized sample without stabilizer, RT: room temperature).

* The FTA card is a cotton-based cellulose matrix containing chemicals that lyse cells, denature proteins and protect DNA; $0.5 \mathrm{ml}$ of homogenized solution was dispatched directly onto the card.

Figure 2: Quantity and quality of DNA extracted from human fecal samples.

(A) DNA yield, expressed as $\mathrm{ng} / \mu 1$. (B) A 260/280 $\mathrm{nm}$ ratio indicative of the presence or absence of phenol, solvent and protein-type contaminants in the DNA extract. The green range indicates a ratio between 1.7 and 2.0, which here defines an optimal DNA quality.

Figure 3: Mean dispersion using Bray-Curtis distances among technological replicates prior (Sc) and after (Dc) homogenization across 15 fecal samples.

The red dotted line indicates equality of dispersion among 'Sc' and 'Dc' samples.

Figure 4: Summary of community shifts in response to stabilizing solutions over a 15-day storage period.

(A) Bray-Curtis distance towards the reference for each patient grouped by stabilizing solution.

Median and 5th-to-95th percentile ranges are shown for both interaliquot and interpatient variability.

(B) A pairwise paired Wilcoxon test was performed to compare solutions with each other.

Significance is shown as follows:

(*) indicates $\mathrm{fdr}<0.05,(* *)$ indicates $\mathrm{fdr}<0.01$, $(* * *)$ indicates $\mathrm{fdr}<0.001$.

Figure 5: Differentially abundant bacterial phyla between samples and their references among the 10 tested DNA stabilizing solutions. 
The median log2-fold change of average profiles is shown with the significance according the corresponding paired Wilcoxon test. $(*)$ indicates $\mathrm{fdr}<0.05,(* *)$ indicates $\mathrm{fdr}<0.01,(* * *)$ indicates $\mathrm{fdr}<0.001$.

\section{SUPPLEMENTARY MATERIAL}

Supplementary Figure S1: Effects of storage conditions on alpha diversity with respect to the observed richness and Shannon index.

\section{Supplementary Figure S2: Mean dispersion among technological replicates prior to (Sc)} and after (Dc) homogenization across 15 fecal samples.

(A) Mean dispersion using the Jaccard distance. (B) Mean dispersion using the Aitchison distance. The red dotted lines indicate the equality of dispersion among 'Sc' and 'Dc' samples.

\section{Supplementary Figure S3: Summary of community shifts in response to stabilizing} solutions over a 15-day storage period.

(A) Jaccard distance towards the reference for each participant, grouped by stabilizing solution, and the median and 5th-to-95th percentile range are shown for both interaliquot and interpatient variability. (B) A pairwise paired Wilcoxon test was performed to compare solutions with each other. Significance is shown as follows: $\left(^{*}\right)$ indicates $\mathrm{fdr}<0.05$, (**) indicates $\operatorname{fdr}<0.01,(* * *)$ indicates $\operatorname{fdr}<0.001$. 
Supplementary Figure S4: Summary of community shifts in response to stabilizing solutions over a 15-day storage period.

(A) The Aitchison distance towards the reference for each participant, grouped by stabilizing solution, median and 5th-to-95th percentile range are shown for both interaliquot and interpatient variability. (B) A pairwise paired Wilcoxon test was performed to compare solutions with each other. Significance is shown as follows: $(*)$ indicates $\mathrm{fdr}<0.05,(* *)$ indicates $\mathrm{fdr}<0.01,(* * *)$ indicates $\mathrm{fdr}<0.001$.

\section{Supplementary Figure S5: Hierarchical clustering based on the Bray-Curtis distance} matrix of all samples in the data set.

The first 4 digits of the sample IDs refer to the biological origin of the fecal sample, and the remaining digits refer to the storage conditions (i.e., Sc, Dc, D, S or stabilizing solutions). Technological replicates clustered together, except for SSO1Sc1-3, SSO2Sc1-3 and SSO4Dc3-3 (shown in red), which were excluded from downstream analysis.

\section{Supplementary Figure S6: Differentially abundant bacterial genera among samples and}

\section{their references among 10 tested DNA stabilizing solutions.}

The median log2-fold change between average profiles and significance of the corresponding paired Wilcoxon test are shown. $(*)$ indicates $\mathrm{fdr}<0.05,(* *)$ indicates $\mathrm{fdr}<0.01,(* * *)$ indicates $\mathrm{fdr}<0.001$.

\section{Supplementary Table 1: Phenotypic characteristics of the 50 most abundant genera}

\section{recovered following a 15-day storage period.}

NEG: negative Gram strain; POS: positive Gram strain; VAR: variable Gram stain; ANAER-ST: strict anaerobic; AER-ST: strict aerobic; FAC: facultative anaerobic; MICRO-AE: microaerophile. 
* Proportion (\%): average relative abundance of each genera within the overall dataset.

\section{References}

1. Lozupone CA, Stombaugh JI, Gordon JI, Jansson JK, Knight R. Diversity, stability and resilience of the human gut microbiota. Nature. 2012;489(7415):220-230. doi: 10.1038/nature11550.

2. Sekirov I, Russell SL, Antunes LCM, Finlay BB. Gut microbiota in health and disease. Physiological reviews. 2010;90(3):859-904. doi: 10.1152/physrev.00045.2009.

3. Sommer F, Bäckhed F. The gut microbiota-masters of host development and physiology. Nature Reviews Microbiology. 2013;11(4):227-238. doi:

\subsection{8/nrmicro2974.}

4. Ley RE, Turnbaugh PJ, Klein S, Gordon JI. Microbial ecology: human gut microbes associated with obesity. Nature. 2006;444(7122):1022-1023. doi: 10.1038/4441022a.

5. Sartor RB. Microbial influences in inflammatory bowel diseases. Gastroenterology. 2008;134(2):577-594. doi: 10.1053/j.gastro.2007.11.059.

6. Wen L, Ley RE, Volchkov PY, Stranges PB, Avanesyan L, Stonebraker AC, et al. Innate immunity and intestinal microbiota in the development of Type 1 diabetes. Nature. 2008;455(7216):1109-1113. doi: 10.1038/nature07336.

7. Louis P, Hold GL, Flint HJ. The gut microbiota, bacterial metabolites and colorectal cancer. Nature reviews microbiology. 2014;12(10):661-672. doi:

\section{$\underline{10.1038 / \text { nrmicro3344. }}$.}


8. Cryan JF, Dinan TG. Mind-altering microorganisms: the impact of the gut microbiota on brain and behaviour. Nature reviews neuroscience. 2012;13(10):701-712. doi: $\underline{10.1038 / \mathrm{nrn} 3346 .}$

9. Nguyen TLA, Vieira-Silva S, Liston A, Raes J. How informative is the mouse for human gut microbiota research?. Disease models \& mechanisms. 2015;8(1):1-16. doi: 10.1242/dmm.017400.

10. Hornung BV, Zwittink RD, Kuijper EJ. Issues and current standards of controls in microbiome research. FEMS microbiology ecology. 2019;95(5):fiz045. doi:

\section{$\underline{10.1093 / \mathrm{femsec} / \mathrm{fiz} 045}$.}

11. Costea PI, Zeller G, Sunagawa S, Pelletier E, Alberti A, Levenez F, et al. Towards standards for human fecal sample processing in metagenomic studies. Nature biotechnology. 2017;35(11):1069-1076. doi: 10.1038/nbt.3960.

12. Kim D, Hofstaedter C E, Zhao C, Mattei L, Tanes C, Clarke E, et al. Optimizing methods and dodging pitfalls in microbiome research. Microbiome. 2017;5(1):1-14. doi: $10.1186 / \mathrm{s} 40168-017-0267-5$.

13. Conrads G, Abdelbary MM. Challenges of next-generation sequencing targeting anaerobes. Anaerobe. 2019;58:47-52.doi: 10.1016/j.anaerobe.2019.02.006.

14. Nechvatal JM, Ram JL, Basson MD, Namprachan P, Niec SR, Badsha KZ. Fecal collection, ambient preservation, and DNA extraction for PCR amplification of bacterial and human markers from human feces. Journal of microbiological methods. 2008;72(2):124-132. doi: 10.1016/j.mimet.2007.11.007.

15. Roesch LF, Casella G, Simell O, Krischer J, Wasserfall CH, Schatz D. Influence of fecal sample storage on bacterial community diversity. The open microbiology journal. 2009;3:40. doi: 10.2174/1874285800903010040. 
16. Lauber CL, Zhou N, Gordon JI, Knight R, Fierer N. Effect of storage conditions on the assessment of bacterial community structure in soil and human-associated samples. FEMS microbiology letters. 2010;307(1):80-86. doi: $10.1111 / \mathrm{j} .1574-$ 6968.2010.01965.x.

17. Cardona S, Eck A, Cassellas M, Gallart M, Alastrue C, Dore J, et al. Storage conditions of intestinal microbiota matter in metagenomic analysis. BMC microbiology. 2012;12(1):1-8. doi: 10.1186/1471-2180-12-158.

18. Choo JM, Leong LE, Rogers GB. Sample storage conditions significantly influence faecal microbiome profiles. Scientific reports. 2015;5(1):1-10. doi: 10.1038/srep16350.

19. Guo Y, Li SH, Kuang YS, He JR, Lu JH, Luo BJ. Effect of short-term room temperature storage on the microbial community in infant fecal samples. Scientific reports. 2016;6-26648. doi: 10.1038/srep26648.

20. Gorzelak MA, Gill SK, Tasnim N, Ahmadi-Vand Z, Jay M, Gibson DL. Methods for improving human gut microbiome data by reducing variability through sample processing and storage of stool. PloS one. 2015;10(8):e0134802. doi:

\subsection{1/journal.pone.0134802.}

21. Hickl O, Heintz-Buschart A, Trautwein-Schult A, Hercog R, Bork P, Wilmes P, Becher D. Sample preservation and storage significantly impact taxonomic and functional profiles in metaproteomics studies of the human gut microbiome. Microorganisms. 2019;7(9):367. doi: 10.3390/microorganisms7090367.

22. Chen CC, Wu WK, Chang CM, Panyod S, Lu TP, Liou JM, et al. Comparison of DNA stabilizers and storage conditions on preserving fecal microbiota profiles. Journal of the Formosan Medical Association. 2020. doi: 10.1016/j.jfma.2020.01.013. 
23. Wu WK, Chen CC, Panyod S, Chen RA, Wu MS, Sheen LY, Chang SC. Optimization of fecal sample processing for microbiome study—The journey from bathroom to bench. Journal of the Formosan Medical Association. 2019;118(2):545-555. doi: 10.1016/i.jfma.2018.02.005.

24. Thomas V, Clark J, Doré J. Fecal microbiota analysis: an overview of sample collection methods and sequencing strategies. Future microbiology. 2015;10(9):14851504. doi: $\underline{10.2217 / \mathrm{fmb} .15 .87 .}$.

25. Hale VL, Tan CL, Knight R, Amato KR. Effect of preservation method on spider monkey (Ateles geoffroyi) fecal microbiota over 8 weeks. Journal of microbiological methods. 2015;113:16-26. doi: 10.1016/j.mimet.2015.03.021.

26. Sinha R, Chen J, Amir A, Vogtmann E, Shi J, Inman KS, et al. Collecting fecal samples for microbiome analyses in epidemiology studies. Cancer Epidemiology and Prevention Biomarkers. 2016;25(2):407-416. doi: 10.1158/1055-9965.EPI-15-0951.

27. Song SJ, Amir A, Metcalf JL, Amato KR, Xu ZZ, Humphrey G, Knight R. Preservation methods differ in fecal microbiome stability, affecting suitability for field studies. MSystems. 2016;1(3):e00021-16. doi: 10.1128/mSystems.00021-16.

28. Shaw AG, Sim K, Powell E, Cornwell E, Cramer T, McClure ZE, et al. Latitude in sample handling and storage for infant faecal microbiota studies: the elephant in the room?. Microbiome. 2016;4(1):40. doi: 10.1186/s40168-016-0186-X.

29. Voigt AY, Costea PI, Kultima JR, Li SS, Zeller G, Sunagawa S, Bork P. Temporal and technical variability of human gut metagenomes. Genome biology. 2015;16(1):73. doi: $10.1186 / \mathrm{s} 13059-015-0639-8$.

30. Wu GD, Lewis JD, Hoffmann C, Chen YY, Knight R, Bittinger K, et al. Sampling and pyrosequencing methods for characterizing bacterial communities in the human gut 
using 16S sequence tags. BMC microbiology. 2010;10(1):1-14. doi: 10.1186/14712180-10-206.

31. Fouhy F, Deane J, Rea MC, O’Sullivan Ó, Ross RP, O'Callaghan G, et al. The effects of freezing on faecal microbiota as determined using MiSeq sequencing and culturebased investigations. PloS one. 2015;10(3):e0119355. doi:

\section{$\underline{10.1371 / \text { journal.pone.0119355. }}$}

32. Bahl MI, Bergström A, Licht TR. Freezing fecal samples prior to DNA extraction affects the Firmicutes to Bacteroidetes ratio determined by downstream quantitative PCR analysis. FEMS microbiology letters. 2012;329(2):193-197. doi: 10.1111/j.15746968.2012.02523.x.

33. Carroll IM, Ringel-Kulka T, Siddle JP, Klaenhammer TR, Ringel, Y. Characterization of the fecal microbiota using high-throughput sequencing reveals a stable microbial community during storage. PloS one. 2012;7(10):e46953. doi: 10.1371/journal.pone.0046953.

34. Illumina. Preparing 16S Ribosomal RNA Gene Amplicons for the Illumina MiSeq System.https://support.illumina.com/documents/documentation/chemistry_documentati on/16s/16s-metagenomic-library-prep-guide-15044223-b.pdf. Accessed 2018.

35. Bolyen E, Rideout JR, Dillon MR, Bokulich NA, Abnet CC, Al-Ghalith GA, et al. Reproducible, interactive, scalable and extensible microbiome data science using QIIME 2. Nature biotechnology. 2019;37(8):852-857. doi: 10.1038/s41587-019-0209$\underline{9}$.

36. Callahan BJ, McMurdie PJ, Rosen MJ, Han AW, Johnson AJA, Holmes SP. DADA2: high-resolution sample inference from Illumina amplicon data. Nature methods. 2016;13(7):581-583. doi: 10.1038/nmeth.3869. 
37. Wood D E, Salzberg SL. Kraken: ultrafast metagenomic sequence classification using exact alignments. Genome biology. 2014;15(3):1-12. doi: 10.1186/gb-2014-15-3-r46.

38. McMurdie PJ, Holmes S. phyloseq: an R package for reproducible interactive analysis and graphics of microbiome census data. PloS one. 2013;8(4):e61217. doi: 10.1371/journal.pone.0061217.

39. Gloor GB, Macklaim JM., Pawlowsky-Glahn V, Egozcue JJ. Microbiome datasets are compositional: and this is not optional. Frontiers in microbiology. 2017;8:2224. doi: $\underline{10.3389 / \text { fmicb.2017.02224. }}$

40. Schmaljohn AL, McClain D. Alphaviruses (Togaviridae) and Flaviviruses (Flaviviridae). In: Baron S, editor. Medical Microbiology. $4^{\text {th }}$ ed. Galveston (TX): University of Texas Medical Branch at Galveston. 1996. Chapter 54.

41. Brenner DJ, Staley JT, Krieg NR. Classification of procaryotic organisms and the concept of bacterial speciation. In: John Wiley \& Sons, Inc., in association with Bergey's Manual Trust. Bergey’s Manual «of Systematic Bacteriology. 2005. P. 2732. doi: $10.1002 / 9781118960608 . b m 00006$.

42. Lowy F. Bacterial classification, structure and function. In: Columbia University. Lecture notes. 2009. http://www.columbia.edu/itc/hs/medical/pathophys/id/2009/introNotes.pdf. Accessed 2019.

43. Parte AC. LPSN-List of Prokaryotic names with Standing in Nomenclature (bacterio. net), 20 years on. Int J Syst Evol Microbiol. 2018;68(6):1825-1829. doi: $\underline{10.1099 / \mathrm{ijsem} .0 .002786 .}$.

44. Abrahamson M, Hooker E, Ajami NJ, Petrosino JF, Orwoll ES. Successful collection of stool samples for microbiome analyses from a large community-based population of 
elderly men. Contemporary clinical trials communications. 2017;7:158-162. doi: 10.1016/j.conctc.2017.07.002.

721

722

45. Williams GM, Leary SD, Ajami NJ, Chipper Keating S, Petrosin JF, Hamilton-Shield JP, Gillespie KM. Gut microbiome analysis by post: Evaluation of the optimal method to collect stool samples from infants within a national cohort study. PloS one. 2019;14(6):e0216557. doi: 10.1371/journal.pone.0216557.

46. Flores R, Shi J, Yu G, Ma B, Ravel J, Goedert JJ, Sinha R. Collection media and delayed freezing effects on microbial composition of human stool. Microbiome. 2015;3(1):33. doi: 10.1186/s40168-015-0092-7.

47. Dominianni C, Wu J, Hayes RB, Ahn J. Comparison of methods for fecal microbiome biospecimen collection. BMC microbiology. 2014;14(1):103. doi: 10.1186/14712180-14-103.

48. Vogtmann E, Chen J, Amir A, Shi J, Abnet CC, Nelson H, et al. Comparison of collection methods for fecal samples in microbiome studies. American journal of epidemiology. 2017;185(2):115-123. doi: 10.1093/aje/kww177.

49. Vogtmann E, Chen J, Kibriya MG, Chen Y, Islam T, Eunes M, et al. Comparison of fecal collection methods for microbiota studies in Bangladesh. Appl. Environ. Microbiol. 2017;83(10):e00361-17. doi: 10.1128/AEM.00361-17.

50. Salonen A, Nikkilä J, Jalanka-Tuovinen J, Immonen O, Rajilić-Stojanović M, Kekkonen RA, et al. Comparative analysis of fecal DNA extraction methods with phylogenetic microarray: effective recovery of bacterial and archaeal DNA using mechanical cell lysis. Journal of microbiological methods. 2010;81(2):127-134. doi: 10.1016/j.mimet.2010.02.007. 

demonstrate a bias in microbiome composition by cell wall structure. Scientific reports. 2019;9(1):1-8. doi: 10.1038/s41598-019-53183-5.

745 


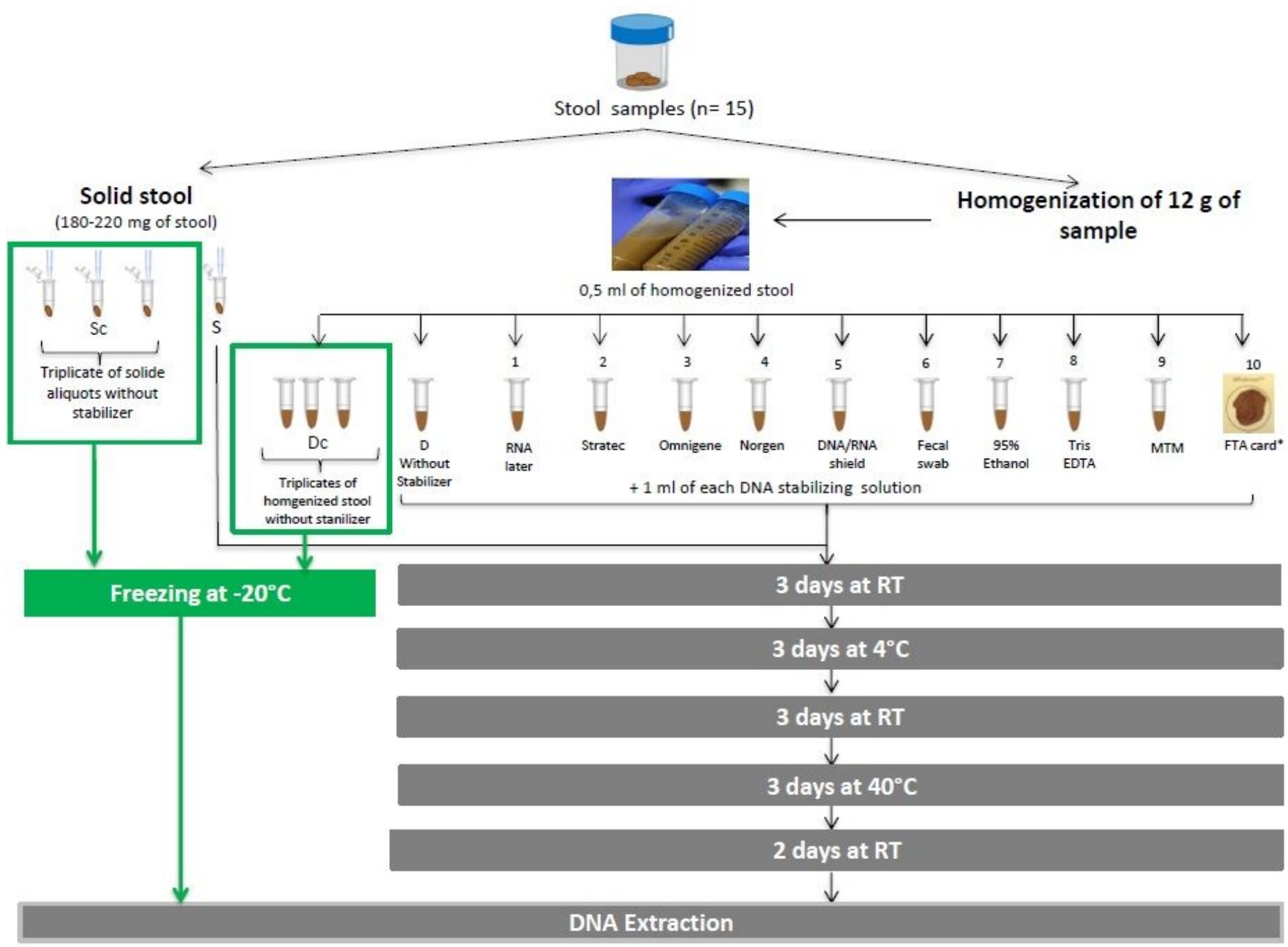

\section{Figure 1}

Illustration of the experimental protocol. Evaluation of ten commercial DNA stabilizing solutions for the storage of fecal samples. (Sc: frozen solid sample, Dc: frozen homogenized sample, S: solid sample without stabilizer, D: homogenized sample without stabilizer, RT: room temperature). * The FTA card is a cotton-based cellulose matrix containing chemicals that lyse cells, denature proteins and protect DNA; 0.5 $\mathrm{ml}$ of homogenized solution was dispatched directly onto the card. 

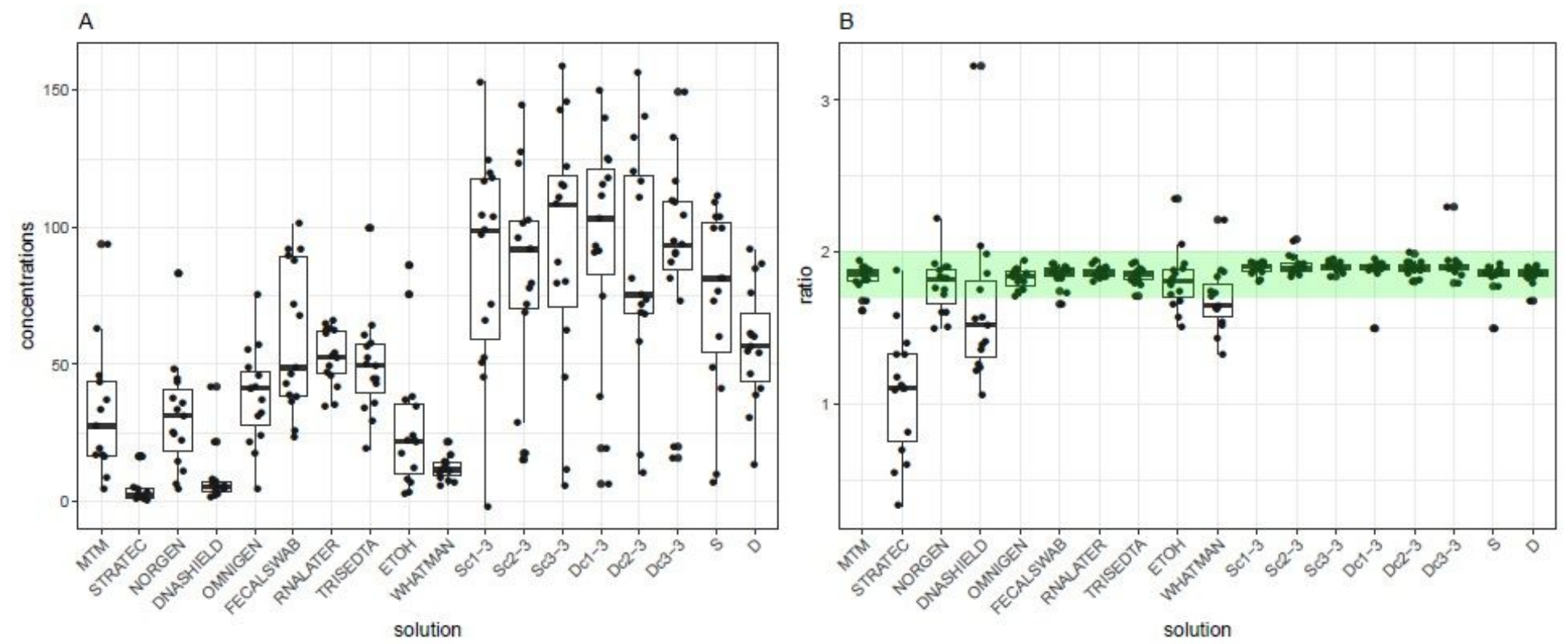

Figure 2

Quantity and quality of DNA extracted from human fecal samples. (A) DNA yield, expressed as $n g / \mu l$. (B) A 260/280 $\mathrm{nm}$ ratio indicative of the presence or absence of phenol, solvent and protein-type contaminants in the DNA extract. The green range indicates a ratio between 1.7 and 2.0, which here defines an optimal DNA quality. 


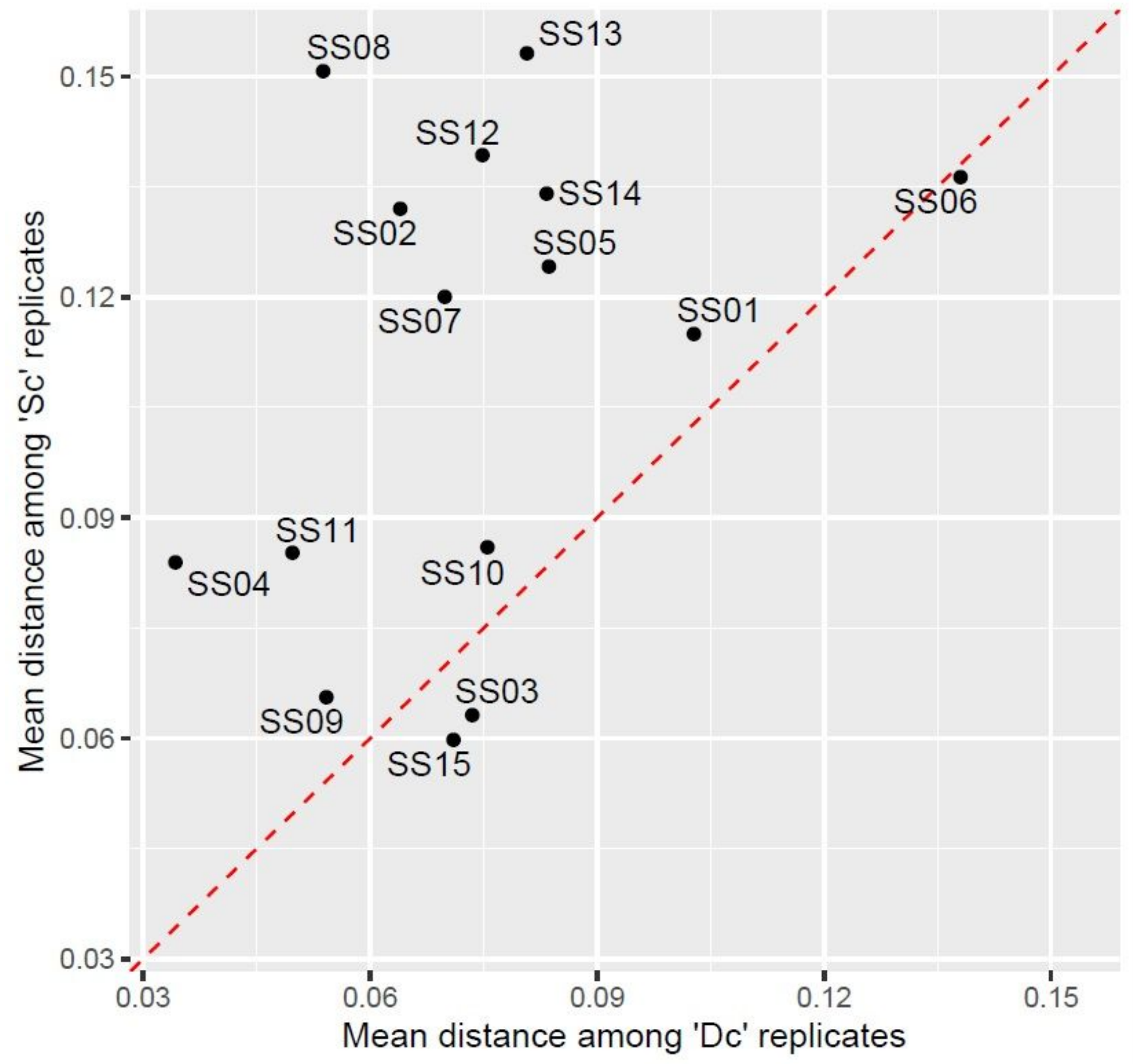

Figure 3

Figure 3: Mean dispersion using Bray-Curtis distances among technological replicates prior (Sc) and after (Dc) homogenization across 15 fecal samples. The red dotted line indicates equality of dispersion among 'Sc' and 'Dc' samples. 


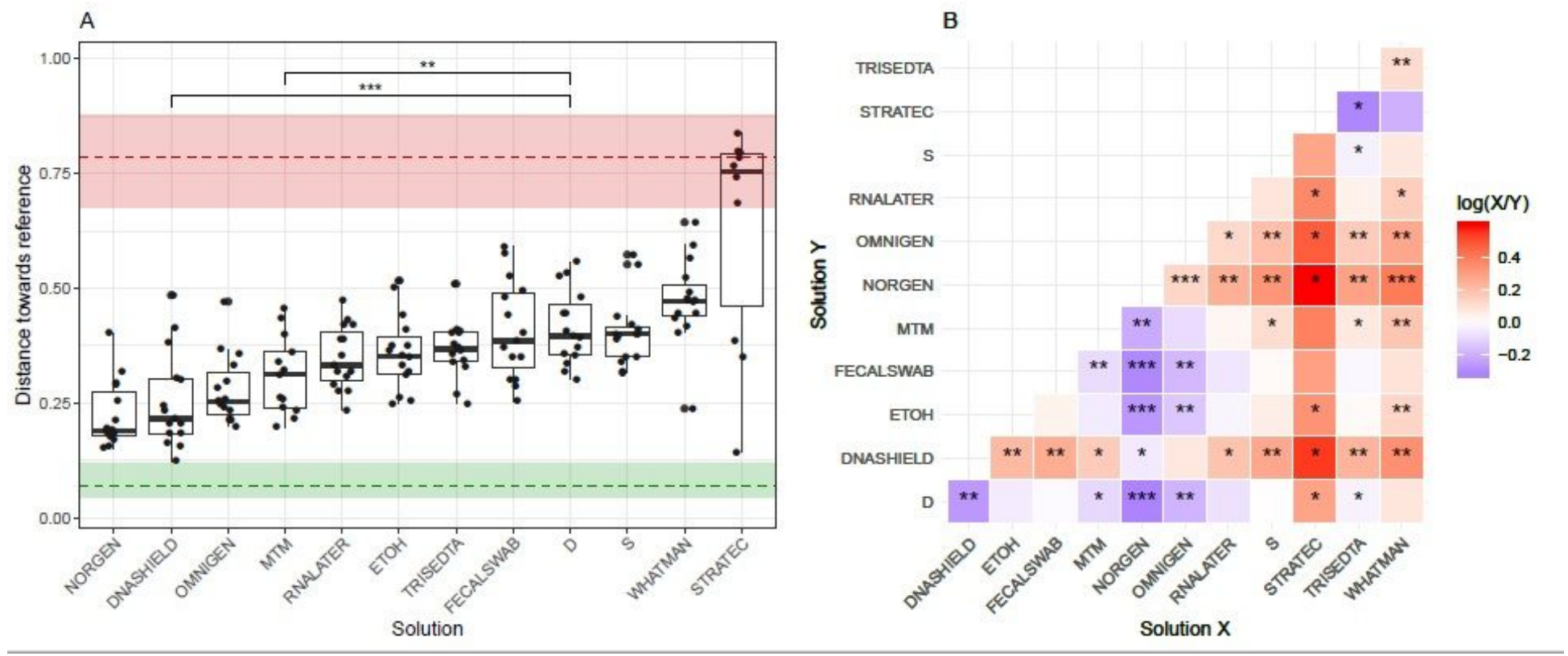

\section{Figure 4}

Summary of community shifts in response to stabilizing solutions over a 15-day storage period. (A) BrayCurtis distance towards the reference for each patient grouped by stabilizing solution. Median and 5th-to95th percentile ranges are shown for both interaliquot and interpatient variability. (B) A pairwise paired Wilcoxon test was performed to compare solutions with each other. Significance is shown as follows: (*) indicates $\mathrm{fdr}<0.05$, $\left({ }^{* *}\right)$ indicates $\mathrm{fdr}<0.01,\left({ }^{* * *}\right)$ indicates $\mathrm{fdr}<0.001$. 


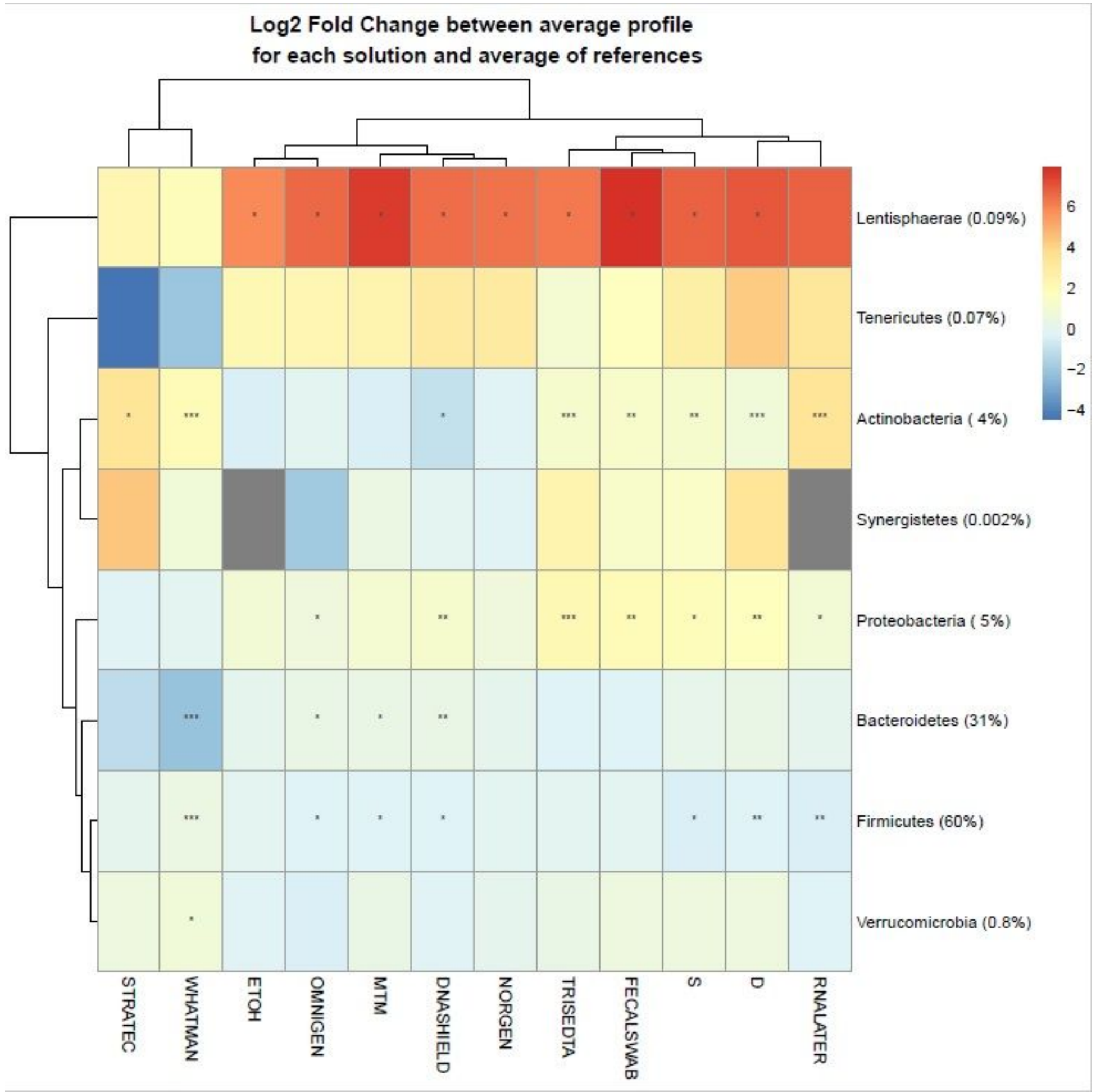

Figure 5

Differentially abundant bacterial phyla between samples and their references among the 10 tested DNA stabilizing solutions. The median log2-fold change of average profiles is shown with the significance

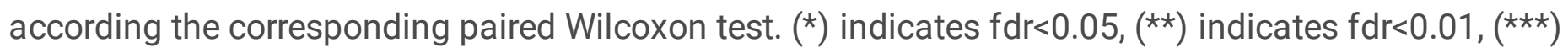
indicates $\mathrm{fdr}<0.001$.

\section{Supplementary Files}


This is a list of supplementary files associated with this preprint. Click to download.

- FigureS1.pdf

- FigureS2.pdf

- FigureS3.pdf

- Figures4.pdf

- Figures5.pdf

- Tables6.pdf 